\title{
Effect of feeding on jugular venous blood flow in the normal newborn infant
}

\author{
P R F DEAR
}

Neonatal Research Group, The London Hospital Medical College

SUMMARY Jugular venous occlusion plethysmography was used to measure jugular blood flow in 47 infants; this was done at varying times after the end of a feed. Jugular blood flow was found to be lower, by about $30 \%$, during the first postprandial half hour than at any other time up to 2 hours postprandially. In 2 infants longitudinal studies were performed in relation to several feeds, and postprandial reductions in blood flow of a similar magnitude were found. Aspects of the venous occlusion technique are discussed. It is concluded that feeding in healthy neonates is associated with a subsequent reduction in jugular blood flow, and possible mechanisms for this phenomenon are discussed.

Cross et al..$^{1}$ found, in a premature infant, that there was an increase in aural temperature after a feed compared with deep body temperature at other sites - such as the rectum and oesophagus. The aural temperature was measured with a neonatal zerogradient aural thermometer (Muirhead Ltd). It was argued that the differential rise in temperature at the aural site was probably due to an increase in brain temperature, the thermistor recording the aural temperature being separated from the temporal lobe by only a thin plate of bone. Later that year Cross et $a .^{2}$ reported that in a number of infants an increase in head temperature could be observed after a feed by means of infrared thermography. The temperature began to rise some 30 to 45 minutes after the end of the feed and remained high for about 60 minutes.

In both studies it was suggested that the rise in temperature might be explained either by an increase in brain heat production, or by a decrease in heat transport from the brain, or a combination of these two factors. There is evidence that there is a general postprandial increase in body metabolism as shown by an increase in total body oxygen consumption, ${ }^{3}$ but the magnitude of this increase is insufficient to account for the estimated rise in brain temperature, even if all the metabolic increase takes place in brain tissue (J K Stothers, 1978, personal communication). It seems probable therefore that there is an element of decreased heat transport away from the brain-that

Department of Paediatrics, New Addenbrooke's Hospital, Cambridge

P R F DEAR, senior registrar is, a diminished brain blood flow. This is an important suggestion which offers a sinister alternative explanation for such postprandial phenomena as the irregularities of breathing often associated with bolus feeding in preterm infants, traditionally attributed to the effect of a distended stomach splinting the thoracic contents. ${ }^{4}$

Before venous occlusion plethysmography was introduced for estimating jugular venous blood flow, ${ }^{5-6}$ there had been no ethical way of assessing cranial blood flow in the normal infant. The method was devised specifically for the study of postprandial changes in cranial blood flow, and this paper reports the first results.

\section{Method}

Technique. A 4-strand mercury-in-Silastic strain gauge was used to record the rate of increase of the occipitofrontal circumference (OFC) of the compliant neonatal cranium after digital occlusion of the internal jugular veins in the supraclavicular region of the neck (Fig. 1). Changes in the length of the gauge cause proportional changes in its electrical resistance. These were measured by incorporating the strain gauge as one of the arms of a Wheatstone bridge. The output of the bridge was recorded on a Hewlitt Packard pen-recorder (7754A) through a preamplifier (Hewlitt Packard Bioelectric 8811A). Fig. 2 shows a recording of two jugular occlusions and a simultaneous electrocardiogram. It can be seen that the infant's cranium pulsates in time with the heart, and that when the jugular veins are occluded there is a rapid pulsatile increase in OFC, the rate of which 


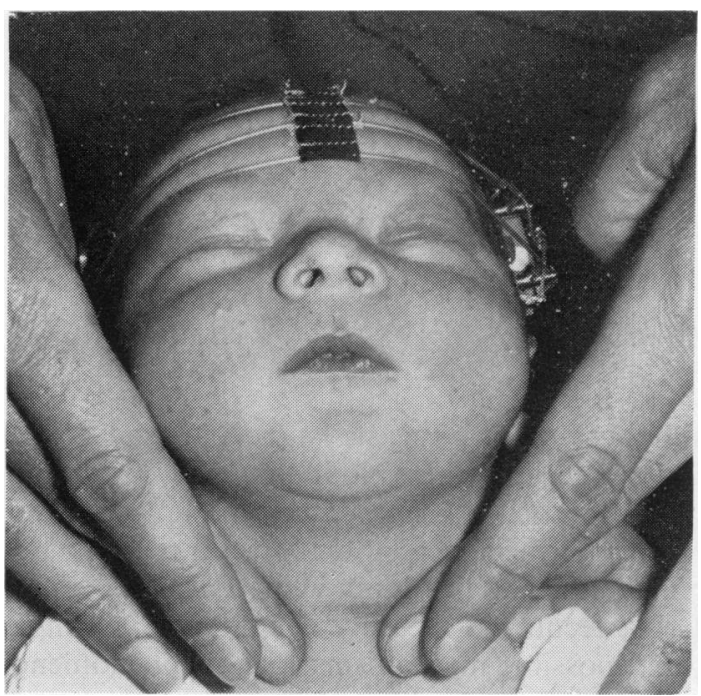

Fig. 1 Digital compression of the jugular veins. The pressure used is no greater than that during clinical examination of the neck as the veins are superficial, thin-walled, low pressure vessels. The infant is wearing a mercury-in-Silastic strain gauge.

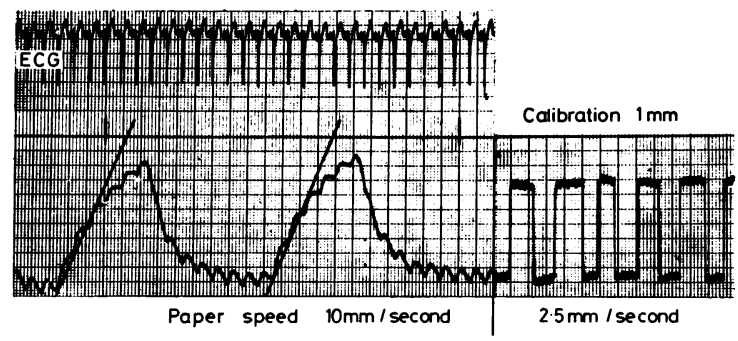

Fig. 2 Two typical inflow curves representing increase in cranial circumference with time. A series of $1 \mathrm{~mm}$ calibration deflections is shown on the right of this recording. Construction lines have been added to illustrate the manner in which the rate of increase of circumference during the first postocclusion cardiac cycle is estimated. A simulianeously recorded ECG is shown.

declines with time. The output of the gauge was calibrated by recording the pen deflection resulting from a known stretch of the gauge. Calibration was conducted in situ on the head by means of an incorporated pneumatic calibrator, worked by a syringe through a length of flexible tubing, designed to effect a $1 \mathrm{~mm}$ extension of the gauge. All measurements were conducted with the infant lying down.
Analysis of records. When the jugular veins are occluded blood begins to accumulate in the cranium and the OFC starts to increase. At the instant of vein occlusion the rate of increase reflects the preocclusion flow in the jugular veins, but as the intracranial pressure rises, so flow in unoccluded vessels-such as the vertebral veins-is recruited and the rate of increase in OFC gradually decelerates, until the entire cranial blood flow is accommodated by unoccluded veins and no further change occurs. The best estimate of jugular venous blood flow is therefore obtained from the rate of OFC increase immediately after vein occlusion. In this study, the mean rate of OFC increase during the first complete cardiac cycle atter occlusion was obtained from the slope of a construction line drawn on the trace, as in Fig. 2. Analysis was facilitated if the occlusion was timed to coincide with a pulse wave nadir, and this was easy to achieve with a little practice. With knowledge of the recording paper speed, the calibration deflection, and the OFC measurement, the percentage rate of circumference increase was calculated in $\mathrm{mm} / \mathrm{min}$ per $100 \mathrm{~mm}$ of OFC. This is the form in which the results are presented.

For a single measurement at least 10 inflow curves were obtained in about 10 minutes. A value for rate of circumference increase was obtained for each inflow curve and then the mean and SE was calculated.

Subjects. Most of the data are given in the form of a cross-sectional study on 45 infants measured at varying times after the end of a feed. The infants were selected at random, but the advice of the ward sisters was taken as to which mothers were most likely to understand the nature and importance of the study.

The mean weight of the infants was $3 \cdot 1$ (range $2 \cdot 2$ to $4 \cdot 3) \mathrm{kg}$ and the mean postnatal age was 4 (range 2 to 9) days. Gestational ages ranged from 36 to 41 weeks. 23 babies were girls and 22 boys. The babies were normal, healthy infants, and none had had any respiratory or other illness. All but 4 were breast fed.

In 2 additional infants longitudinal studies were performed in relation to several feeds. Case 1 was a term, 3.45-kg, 3-day-old, breast-fed infant. Pre- and postprandial measurements of jugular blood flow were obtained in relation to two feeds. Case 2 was a term, 3-kg, breast-fed infant, and measurements of blood flow were performed on days $1,2,3,6,7$, and 8 . Both pre- and postprandial blood flow measurements were obtained in relation to six feeds.

All infants were from the postnatal or neonatal wards of this hospital. In each case the procedure was explained in full to one parent or both, and permission obtained. Permission had also been 
obtained from the Hospital Ethical Committee. In most cases one parent (or both) was present for a least part of the time that measurements were taken. No sedation was used in any infant.

Evaluation of the analysis. As part of the assessment of the technique, the strain gauge was applied to a number of dead infants and it was observed that there was sometimes a small fluctuation in the OFC recording during digital compression of the veins due to the effect of skin traction distorting the gauge in the temporal region. Finger-tip pressure recording in live infants showed that the act of digital compression was complete well within one cardiac cycle, and that any 'compression artefact' would be confined to the first pulse of the inflow curves. The method of analysis was therefore open to the criticism that it might include a 'compression artefact'. The significance of any artefact was examined as follows.

The pulsations on the inflow curves were effectively removed by considering only those points on the curve coinciding with the same phase of the cardiac cycle. When this was done the rate of OFC increase could be shown to decay exponentially with time, as would be expected if blood flow in unoccluded veins increases proportionately with rising intracranial pressure. This exponential relationship permits a mathematical treatment of the curves by which the rate of increase in OFC at the moment of vein occlusion can be derived by extrapolation from points on the curve distal to the first pulsation, and any artefact thus excluded.

Inflow curves in the present study were recorded with the original method of analysis in mind. To facilitate this the first two or three pulses were recorded at high amplification, so that not enough points were obtained for reliable exponential analysis. In order to discover how much the results might be affected by artefact, Milligan and Rahilly compared the two methods of analysis in 10 additional infants (D Milligan and P M Rahilly, 1978, personal communication). Exponential analysis was performed using computer program BMD P3R.? Fig. 6 shows a graphical correlation of circumference increase values obtained by both methods of analysis. The correlation is good $(r=0.82, P<0.01)$ and the standard errors of both techniques are comparable. There is a tendency for the simpler method to overestimate low flows and underestimate high flows.

The venous occlusion method measures jugular vein blood flow and takes no account of that proportion of cranial blood which normally flows in vessels inaccessible to external compression. However a model of cranial haemodynamics has now been proposed which takes account of the outflow curve, recorded after the release of jugular vein occlusion, in order to estimate the total cranial flow. ${ }^{6}$ The average contribution to total venous drainage by unoccluded channels was estimated to be in the region of $15 \%$, and the correlation between total cranial blood flow and jugular vein blood flow was found to be very good, suggesting that jugular blood flow is a reliable index of total cranial blood flow.

Conversion of circumference change to volume change. It can be shown that the percentage change in volume of the skull is approximately 3 times the percentage change in OFC, ${ }^{6}$ and this relationship can be used to convert the recorded rate of increase in OFC in $\mathrm{mm} / \mathrm{min}$ per $100 \mathrm{~mm}$ OFC, to jugular blood flow in $\mathrm{ml} / \mathrm{min}$ per $100 \mathrm{ml}$ of cranial volume. Cranial volume was estimated from a power curve relating cranial volume to $\mathrm{OFC}$, constructed from

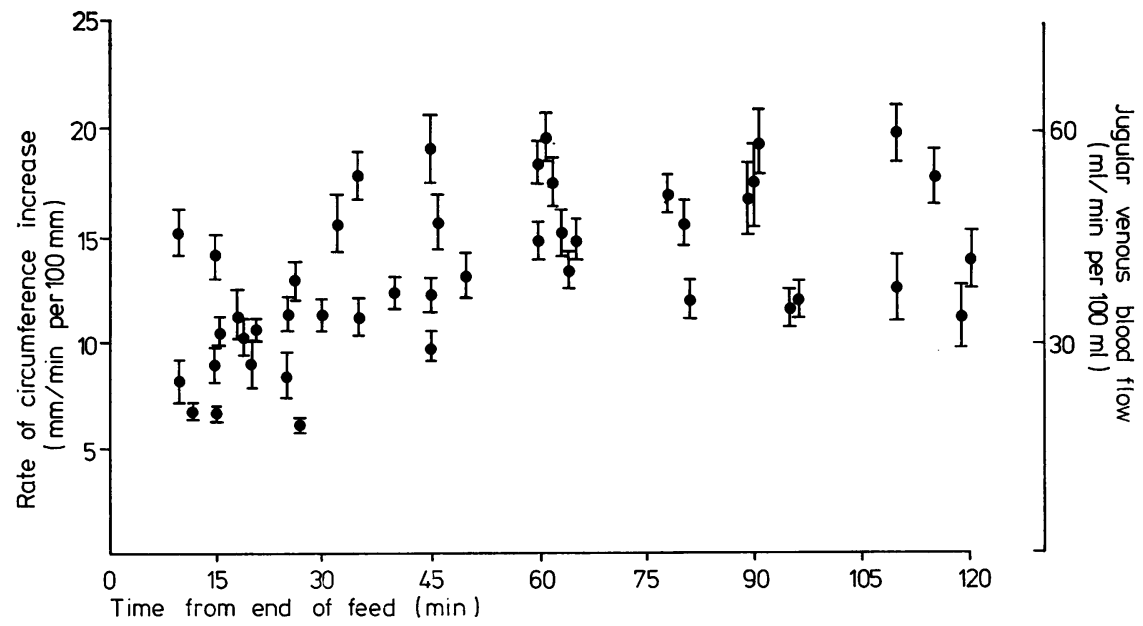

Fig. 3 Rates of increase in cranial circumference for 45 infants, measured at varying times after the end of a feed. The scale on the right of the graph shows derived values for jugular venous blood flow. The bars represent $2 S E$ on each side of the mean. 
the data of Buda et al..$^{9}$ The actual relationship was: Cranial volume $(\mathrm{ml})=\mathrm{OFC}^{3}(\mathrm{~cm}) / 89$

Fig. 3 carries an additional $y$-axis giving derived jugular blood flow in $\mathrm{ml} / \mathrm{min}$ per $100 \mathrm{ml}$ cranial volume.

\section{Results}

Fig. 3 shows rates of increase in the cranial circumference for 45 infants measured at varying times within 2 hours after the end of a feed. Mean rate of circumference increase for infants measured within 30 minutes after the end of a feed was $10 \mathrm{~mm} / \mathrm{min}$ per $100 \mathrm{~mm}$ OFC (SE $0.6 \mathrm{~mm} / \mathrm{min}$ per $100 \mathrm{~mm})$, and that for infants measured more than $\mathbf{3 0}$ minutes after the end of a feed was $14.3 \mathrm{~mm} / \mathrm{min}$ per $100 \mathrm{~mm}$ OFC (SE $0.5 \mathrm{~mm} / \mathrm{min}$ per $100 \mathrm{~mm}$ ). This difference was significant (Wilcoxon's sum of ranks test, $Z 4 \cdot 45$, $P<0 \cdot 002)$. There were no significant differences between boys and girls. The fact that only four infants in the study were bottle fed prevented any valid comparison between bottle and breast feeding.

Fig. 4 shows circumference increase values in Case 2. On the first feed of the first day of life, the difference in pre- and postfeed values was not significant, but on all other occasions when both pre- and postfeed values were available the postprandial fall in jugular blood flow was significant $(P<0 \cdot 001)$. The magnitude of the maximum fall in flow increased from $24 \%$ on day 1 , to $43 \%$ on day 3 , and $35 \%$ on day 7.

Fig. 5 shows values for increase in circumference in relation to two feeds in Case 1. In both instances the postprandial fall in jugular blood flow was significant $(P<0.002)$, being $40 \%$ at one time and $41 \%$ at the other. The longitudinal studies show that immediate preprandial jugular blood flow values are similar to those found about 2 hours postprandially.
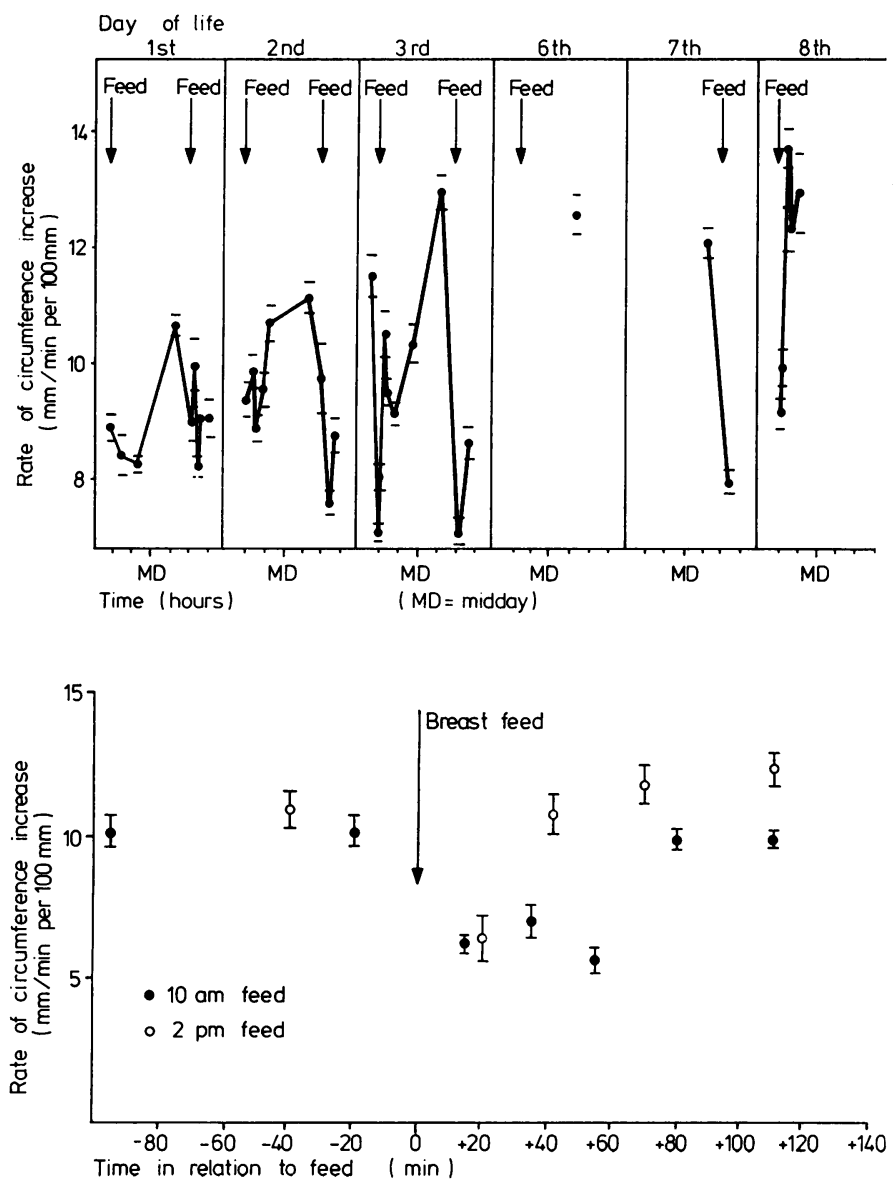

Fig. 4 Serial rate of circumference increase measurements on Case 2 on 6 days of life. The vertical panels represent days as indicated. The divisions on the abscissa are hours. A feed generally took about 20 minutes and arrows indicate the midpoint of each feed. Bars show $1 S E$ on each side of the mean.

Fig. 5 Rate of circumference increase values of Case 1 in relation to two feeds. Each feed took 20 minutes. Negative times are in relation to the start of the feed and positive times in relation to the end of the feed. Vertical bars represent $2 S E$ on each side of the mean. 


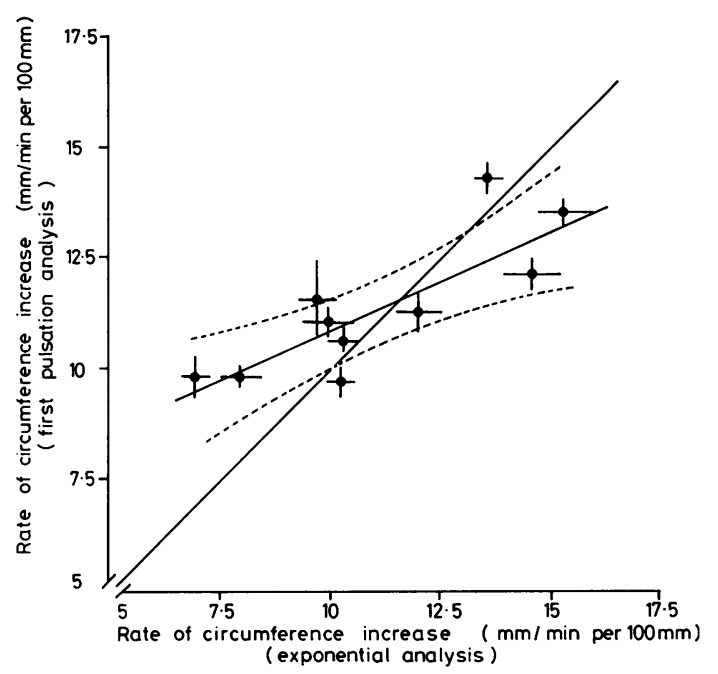

Fig. 6 Correlation between circumference increase values derived by the method of analysis used in this study (first pulsation analysis) and the method using exponential curve fitting minus the first pulsation. The regression line for the two sets of data is shown as well as the line of identity. Bars show 1 SE on each side of the mean. Broken lines show the $95 \%$ confidence limits. The equation for the line of identity is of the form $y=a+b x$, where $a=19.03$ and $b=0.45$. $r=0.82$.

\section{Discussion}

The results of this preliminary study support the suggestion of Cross $e t$ al. ${ }^{1}$ that there is a temporary postprandial fall in brain blood flow in the normal infant. The fall appears to be between about 30 and $40 \%$, with the lowest values found in the first postprandial half hour. Further longitudinal studies (including intrafeed measurements) will be necessary to find the precise timing of the blood flow changes.

Why neonatal cranial blood flow behaves in this way must be a matter for speculation. It does appear to be a peculiarity of the newborn. Rowe et al.,10 using the Kety method, ${ }^{11}$ found no changes in adult cerebral blood flow or cerebral oxygen consumption after a standard meal. Further evidence of a difference in the circulatory response to feeding between newborn babies and adults was provided by Yao et al. ${ }^{12}$ who found an average of $49 \%$ reduction in lower limb blood flow 30 minutes after the end of a feed in term infants. This contrasts with the work of Abramson and Fierst ${ }^{13}$ in which adult lower limb blood flow showed no response to a carbohydrate meal and only a late ( 2 hour) postprandial increase after a protein meal. In relative terms the 'standard meal' of a neonate is considerably larger than the adult equivalent, and it may be that as the circulatory reserve of the newborn is more limited than that of the adult, ${ }^{14}$ a postprandial increase in splanchnic perfusion is met at the expense of other vascular territories, including to some extent the central nervous system. A purely passive reduction in cerebral perfusion seems unlikely however, as there is evidence of well developed peripheral vasomotor responses in the newborn; $;^{15}$ and even if perfusion pressure cannot be fully maintained, there is no reason to believe that neonatal cerebral blood flow is not autoregulated with respect to blood pressure, as it is in the adult. ${ }^{16}$

In addition to the possibility of passive redistribution of the circulating blood volume, there are several factors that are known directly to influence cerebral blood flow, in adults at least, which require consideration in the context of postprandial change. The most potent of these influences is carbon dioxide. Increase in $\mathrm{PaCO}_{2}$ is associated with an increase in cerebral blood flow and the converse is true for depressions in $\mathrm{PaCO}_{2}$. Knowledge about the effect of feeding on pulmonary ventilation and blood-gas status in the neonate is incomplete. Wilkinson and $\mathrm{Yu}^{17}$ studied sick infants fed small volumes of milk $(6 \mathrm{ml} / \mathrm{kg})$ by nasogastric tube and found that $\mathrm{PaCO}_{2}$ was normal 10 minutes after the end of the feed but had fallen 30 minutes later; respiratory rate was raised significantly during the whole 30 -minute study period. There is evidence from using venous occlusion plethysmography that the cranial blood flow of the neonate is at least as sensitive to blood-gas changes as is that of the adult (P M Rahilly, 1978, personal communication), but until the postprandial bloodgas status of the normally-fed healthy infant is established, firm conclusions cannot be made about cranial blood flow changes.

Sleep state has been shown to affect cerebral blood flow in adults, with significantly higher flow in rapid eye movement sleep than in nonrapid eye movement sleep. ${ }^{18}$ There have been similar findings in the normal newborn infant using jugular venous occlusion plethysmography (D Milligan, 1977, personal communication). In view of this the data were re-examined with respect to sleep state; in some infants sleep state had been judged according to traditional observational criteria. It was found that 20 babies were recorded as being in nonrapid eye movement sleep. Of these, the 11 babies measured within $\mathbf{3 0}$ minutes postprandially had a mean jugular blood flow $50 \%$ lower than the 9 measured more than 30 minutes postprandially. Further studies are needed using electroencephalographic criteria for sleep state, but so far the findings suggest that the postprandial fall in blood flow is not due to differences in sleep state alone. 
It seems reasonable to suggest that a postprandial fall in brain blood flow is without adverse effect in the healthy normal newborn infant, and it is important to realise that what has been observed is a fall in average flow to the cranium which may well be accompanied by a redistribution of flow within the brain itself, perhaps with favouring of areas most vulnerable to ischaemia. It has yet to bz established whether the preterm or sick infant shows the same responses, and whether tube feeding or intravenous feeding has a similar effect. It is perhaps in these areas that the venous occlusion method may produce information of clinical importance at a time when there is considerable debate about infant feeding patterns and regimens with a trend towards greater feeding volumes, especially in the preterm and small for gestational age infant. It seems appropriate to inject a word of caution, and to suggest that the human newborn may not be infinitely adaptable in his physiological responses.

I thank Professor K W Cross for guidance, Dr J K Stothers for help with the preparation of the manuscript, and research nurses Miss R M Warner and Miss N Cullen for their expertise with babies. $\mathrm{Mr}$ T G Barnett designed and built the electronics, and Mr R Shew helped to make the gauges.

The work was partly supported by The Wellcome and Sir Halley-Stewart Trusts.

\section{References}

1 Cross K W, Stothers J K, Stratton D. An indirect estimate of total brain energy metabolism in the newborn infant (abstract). J Physiol (Lond) 1975; 250: 15-6P.

2 Cross K W, Stothers J K, Warner R M, Woodruff R W. The application of thermography to the detection of energy metabolism of the brain in the newborn infant (abstract). J Physiol (Lond) 1975; 252: 44-5P.

3 Stothers J K, Warner $\mathbf{R}$ M. The effect of feeding on neonatal oxygen consumption. Arch Dis Child 1979; 54: 415-20.

4 Barrie H. Effect of feeding on gastric and oesophageal pressures in the newborn. Lancet 1968; ii : 1158-60.
5 Cross K W, Dear P R F, Warner R M, Watling G B. An attempt to measure cerebral blood flow in the newborn infant (abstract). J Physiol (Lond) 1976; 260: 42-3P.

6 Cross K W, Dear P R F, Hathorn M K S et al. An estimation of intracranial blood flow in the newborn infant. J Physiol (Lond) 1979; 289: 329-45.

7 Dixon W J. BMDP biomedical computing programs. Manual P3R. Los Angeles: University of California, 1975

8 Cross K W, Kerslake D McK, Rahilly P M. The ratio of cranial to jugular blood flow in the newborn (abstract). J Physiol (Lond) 1978; 280: 62-3P.

- Buda F B, Reed J C, Rabe E F. Skull volume in infants. Am J Dis Child 1975; 129: 1171-4.

10 Rowe G G, Maxwell G M, Castillo C A, Freeman D J, Crumpton C W. A study in man of cerebral blood flow and cerebral glucose, lactate, and pyruvate metabolism before and after eating. $J$ Clin Invest $1959 ; 38: 2154-8$.

11 Kety S S, Schmidt C F. The determination of cerebral blood flow in man by the use of nitrous oxide in low concentration. Am J Physiol 1945; 143: 53-66.

12 Yao A C, Wallgren C G, Sinha S N, Lind J. Peripheral circulatory response to feeding in the newborn infant. Pediatrics 1971 ; 47: 378-83.

13 Abramson D I, Fierst S M. Peripheral vascular responses in man during digestion. Am J Physiol 1941; 133: 686-93.

14 Wallgren G, Hanson J S, Lind J. Quantitative studies of the human neonatal circulation. III. Observation on the newborn infant's central circulatory responses to moderate hypovolaemia. Acta Paediatr Scand (Suppl) 1967; Supplement 179, 43-54.

15 Moss A J, Duffie E T, Jr, Emmanouilides G. Blood pressure and vasomotor reflexes in the newborn infant. Pediatrics $1963 ; 32$ : 175-9.

16 Agnoli A, Fieschi C, Bozzao L, Battistini N, Prencipe M. Autoregulation of cerebral blood flow. Studies during drug-induced hypertension in normal subjects and in patients with cerebral vascular diseases. Circulation 1968; 38: 900-12.

17 Wilkinson A, Yu V Y H. Immediate effects of feeding on blood gases and some cardiorespiratory functions in ill newborn infants. Lancet 1974 ; i: 1083-5.

18 Risberg J, Gustafson L, Ingvar D H. Regional cerebral blood volume during paradoxical sleep. In: Brock $M$, Fieschi C, Ingvar D H, Lassen N A, Schürmann K, eds. Cerebral blood flow. Berlin: Springer-Verlag, 1969: 101-3.

Correspondence to Dr P R F Dear, Department of Paediatrics, New Addenbrooke's Hospital, Hills Road, Cambridge CB2 2QQ.

Received 11 April 1979 